\section{P26 HOW DOES THE SALFORD LUNG STUDY IN COPD (SLS COPD) PATIENT POPULATION FIT INTO THE GOLD 2017 CLASSIFICATION GRID?}

IJ Vestbo, ${ }^{2}$ Boucot, ${ }^{2} \mathrm{~L}$ Frith, ${ }^{3} \mathrm{~N}$ Diar Bakerly, ${ }^{2} \mathrm{~S}$ Collier, ${ }^{2} \mathrm{DA}$ Leather, ${ }^{3} \mathrm{JM}$ Gibson, ${ }^{1}$ A Woodcock. 'Manchester Academic Health Sciences Centre, The University of Manchester, and University Hospital South Manchester NHS Trust, Manchester, UK; ${ }^{2}$ GSK, Uxbridge, UK; ${ }^{3}$ Salford Royal NHS Foundation Trust, Salford, UK and Manchester Academic Health Sciences Centre, The University of Manchester, Manchester, UK

10.1136/thoraxjnl-2017-210983.168

Background GOLD 2017 separates spirometric staging from the 'ABCD' groupings defined by symptom burden/exacerbation risk. SLS COPD, a 12 month randomised controlled trial, demonstrated the effectiveness and safety of initiating fluticasone furoate/vilanterol versus continuing usual care in COPD patients in UK primary care (Vestbo J et al, New Engl J Med 2016;375:1253-60).

Aim Map SLS COPD patient distribution and treatment patterns to the GOLD 2017 classification grid.

Methods In this post-hoc analysis, patients were classified by GOLD 2017 (groups A-D based on exacerbation frequency history and baseline CAT score) and by the previous spirometry-based GOLD 2007 system (stages 1-4 based on baseline post-BD $\mathrm{FEV}_{1}$ ).

Results Baseline characteristics were (ITT; $n=2799$ ): 49\% $\geq$ 2 moderate/severe exacerbations in past year; 90\% CAT score $\geq 10 ; 14 \%$ on long-acting BD only, $34 \%$ ICS \pm LABA or LAMA, 52\% ICS/LABA+LAMA. The figure shows key data. Distribution by GOLD 2017 was $(n=2796)$ : A 7\%; B 43\%; C 3\%; D 47\% and by GOLD 2007 ( $n=2199$ with spirometry data): no airflow obstruction 12\%; 1 10\%; 2 48\%; 3 24\%; 4 $5 \%$. Around $60 \%$ of patients had mild/moderate airflow obstruction while $90 \%$ were GOLD group B/D due to symptoms and exacerbation risk. GOLD ABCD distribution and treatment patterns in patients without spirometry data were similar to the overall population.

Conclusion These data suggest that COPD exacerbations and symptoms are driving appropriate GP COPD management in routine primary care.

Funding GSK (HZC115151/NCT01551758).

Please refer to page A258 for declarations of interest in relation to abstract P26.

\section{\begin{tabular}{l|l} 
P27 & DEPRIVATION IN THE COPD SALFORD LUNG STUDY
\end{tabular} (SLS) IS ASSOCIATED WITH HIGHER HEALTHCARE COSTS BUT DOES NOT MODERATE THE MAIN OUTCOMES}

${ }^{1} R$ Jones, ${ }^{2} A$ Nicholls, ${ }^{2} D$ Browning, ${ }^{3} \mathrm{~N}$ Diar Bakerly, ${ }^{4} A$ Woodcock, ${ }^{4}$ Jestbo, ${ }^{2} D$ Leather ${ }^{1}$ Peninsula School of Medicine and Dentistry, Plymouth University, Plymouth, UK; ${ }^{2}$ GSK, Brentford, UK; ${ }^{3}$ Salford Royal NHS Foundation Trust, Salford, UK; ${ }^{4}$ Manchester Academic Health Sciences Centre, The University of Manchester and South Manchester University Hospital NHS Foundation Trust, Manchester, UK

\subsection{6/thoraxjnl-2017-210983.169}

Introduction and Objectives The SLS was a pragmatic randomised controlled trial (RCT) of initiating fluticasone furoate/ vilanterol (FF/VI) versus continuing usual care (UC) and aimed to recruit a real life population in a near normal care setting. The 12 month study required only two patient visits and most data was collected using electronic health records. This posthoc sub-study assesses the impact of deprivation on health behaviours, healthcare costs and main/safety outcomes.

Methods Deprivation scores were derived by postcode using the countrywide indices of deprivation (version 2010). The indices were categorised into quintiles. The primary effectiveness endpoint of mean annual rate of moderate or severe

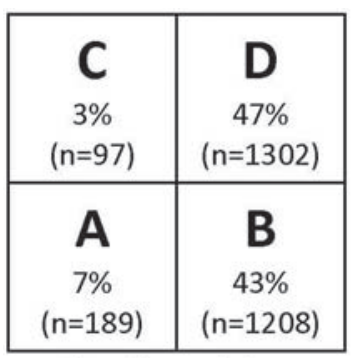

Overall population ( $\mathrm{N}=2799)^{*}$

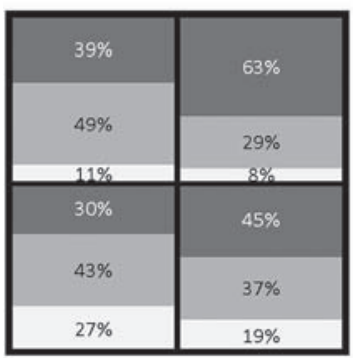

Overall population ( $\mathrm{N}=2799)^{*}$

*CAT data missing for 3 patients

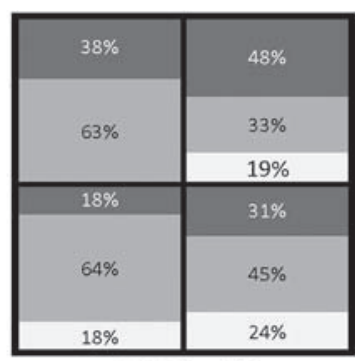

GOLD1; mild obstruction ( $n=229)$

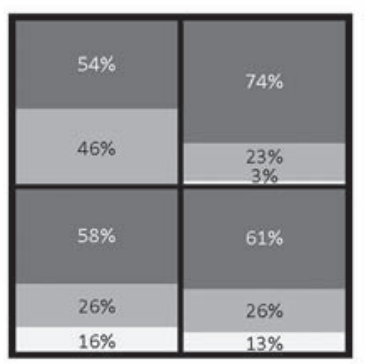

GOLD3; severe obstruction $(n=521)$

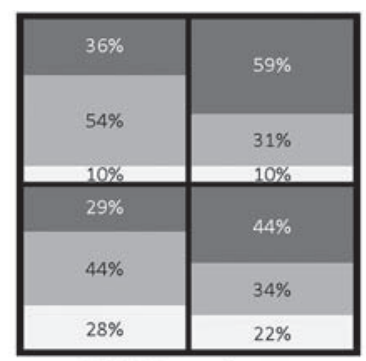

GOLD2; moderate obstruction ( $n=1064$ )

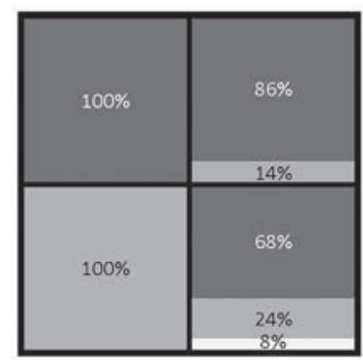

GOLD4; very severe obstruction $(n=117)$

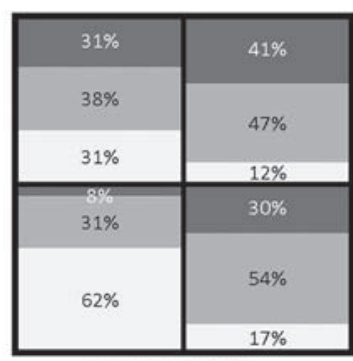

$\mathrm{FEV}_{1} / \mathrm{FVC}>0.7$

"GOLDO"; no obstruction ( $n=268$ )

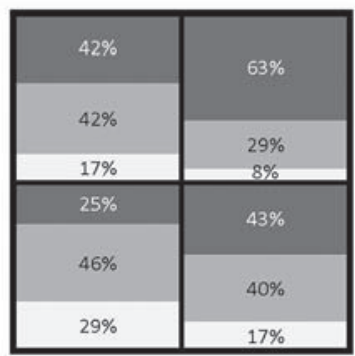

Spirometry data unavailable $(n=600)$

ICS/LABA+LAMA

ICS, ICS/LABA or ICS+LAMA

LABA, LAMA or LABA+LAMA

Abstract P26 Figure 1 SLS COPD patient distribution and baseline treatment by 2017 and 2007 GOLD classification systems. 